\title{
COLETA DE DADOS: AVALIAÇÃO DE UM MODELO PILOTO
}

\author{
Maria Cecília Bueno Jayme Gallani * \\ Roberta Cunha Rodrigues Colombo* \\ Eliane de Araújo Cintra* \\ Sandra Bruneni Rigacci*
}

GALLANI M.C.B.J.; COLOMBO, R.C.R.; CINTRA, E.A.; RIGACCI S.B. Coleta de dados: avaliação de um modelo piloto. Rev.latino-am.enfermagem, Ribeirão Preto, v. 4, n. 2, p. 179-99, julho 1996

Após o desenvolvimento e implementação de um modelo piloto do instrumento de coleta de dados (ICDP) para Unidade Coronária (UCO), tivemos como objetivos avaliar as considerações dos enfermeiros sobre a aplicabilidade deste instrumento e de sua adequação na UCO do HC-UNICAMP bem como levantar subsídios para contribuir na sua reelaboração. A avaliação, realizada através de um questionário entregue a onze enfermeiros que participaram da implementação do ICDP, mostrou-nos que os enfermeiros consideraram válidos o desenvolvimento e implementação do ICDP, interpretado como adequado em forma e conteúdo para pacientes cardiopatas, além de fornecer subsídios para a elaboração do instrumento definitivo de ICD.

UNITERMOS: coronariopatia, unidade de cuidados coronarianos, processos de enfermagem

\section{INTRODUÇÃO}

As características da atividade do enfermeiro determinam a necessidade de sua permanência constante junto ao paciente, tornando-o, dessa forma uma figura chave no "processo terapêutico" (visto aqui como o conjunto de atividades da equipe multidisciplinar interligadas com o objetivo de procurar restaurar 0

\footnotetext{
* Professor. Departamento de Enfermagem - Faculdade de Ciências Médicas - UNICAMP Campinas - SP

${ }^{\star \star}$ Enfermeira. Unidade de Terapia Intensiva do Hospital das Clínicas - UNICAMP - Campinas - SP
} 
equilíbrio do paciente no ciclo saúde-doença). Assim o enfermeiro pode ser considerado como um elemento catalisador e ao mesmo tempo disseminador das informações necessárias a tal processo. Neste sentido, de acordo com $\mathrm{McHUGH}^{13}$ a qualidade da assistência de enfermagem torna-se criticamente importante, não apenas para o paciente, mas também para a instituição, a equipe de saúde e o próprio enfermeiro.

As teorias de enfermagem que surgiram na segunda metade desse século, na definição de CASTELLANOS \& CASTILHO $^{7}$ "têm a finalidade de acompanhar reflexiva e criticamente atividade prática de enfermagem, em esclarecimento, por exemplo, de seu objeto de trabalho (...)", e com isso assegurar a qualidade da atuação do enfermeiro. A maioria delas, independentemente de seu autor ou de sua linha filosófica, traz consigo uma proposta de sistematização do trabalho de enfermagem, ou ainda, de uma Metodologia de Assistência de Enfermagem que, de um modo geral, compreende as seguintes etapas:

1. levantamento de dados do paciente; 2 . identificação de problemas; 3 . estabelecimento de prioridades; 4. elaboração do plano de cuidados a curto, médio e longo prazo; 5. avaliação periódica dos efeitos obtidos frente aos cuidados prestados.

Em nossa instituição, onde o Departamento de Enfermagem tem como filosofia a "prestação de cuidados de enfermagem planejados de acordo com as necessidades específicas do paciente como pessoa, membro de uma família e comunidade", a equipe de enfermagem da Unidade de Terapia Intensiva (UTI)/Unidade Coronária (UCO), constituintes do Núcleo de Assistência Médica Intensiva (NAMI), passou a empregar, desde 1986, a Sistematização da Assistência de Enfermagem, adaptada a partir do modelo teórico proposto por HORTA $^{12}$.

Das seis etapas sugeridas por este modelo. eram empregadas apenas: Histórico de Enfermagem, aqui designado como instrumento de levantamento de dados - ICD (de maneira informal). Prescrição de Enfermagem e Evolução de Enfermagem. Consideramos que o ICD vinha sendo realizado mesmo que sem a existência de um impresso específico, uma vez que, analisando-se, retrospectivamente, os prontuários dos pacientes internados na UTI/UCO no período de 1989 a 1992, identificávamos, no espaço destinado às anotações, um levantamento de dados pertinentes a um ICD. Além disto, observamos que havia uma tendência a caracterização do corpo de dados levantados, de acordo com o tipo de paciente. Esta diferença tornava-se mais evidente quando comparávamos as folhas de admissão dos pacientes internados na UCO com as dos pacientes da UTI geral.

Não obstante, as informações, precisavam ser padronizadas, isto é, havia registros mais detalhados, outros mais sucintos, necessitando de dados que seriam importantes no "processo terapêutico". Não havia, também, homogeneidade na organização deste levantamento, resultando numa história de admissão muitas vezes confusa. 
No estudo feito por ANSELMI et al. ${ }^{1}$ sobre o surgimento, definição do histórico de enfermagem (ou levantamento de dados) e de seu significado na prática, é sugerido um consenso entre os autores pesquisados de que este "é um instrumento a ser utilizado pelo enfermeiro para levantar dados do paciente, e que favorece uma interação efetiva entre ambos".

Estas autoras definem também que, qualquer que seja a Metodologia de Assistência de Enfermagem adotada, a primeira fase constituir-se-á da descrição da situação/problema, exigindo correlação de dados, análise e interpretação, (...) sendo uma etapa fundamental para a operacionalização dos demais passos".

Com base nesses pressupostos e após o desenvolvimento e implementação de um modelo piloto de instrumento de levantamento de dados (ICDP) para UCO, tivemos como objetivos neste trabalho:

1. avaliar as considerações dos enfermeiros sobre a aplicabilidade do ICDP;

2. analisar junto aos enfermeiros a adequação do ICDP para UCO;

3. levantar subsídios que venham a contribuir na reelaboração do ICD definitivo para ser utilizado na assistência de enfermagem dos pacientes da UCO do NAMI do HC-UNICAMP.

\section{MATERIAL E MÉTODOS}

O presente trabalho foi realizado na UCO, integrante do NAM do Hospital de Clínicas da Universidade Estadual de Campinas (UNICAMP), composta por cinco leitos destinados a pacientes cardiopatas, portadores, em sua maioria, de coronariopatias.

Onze enfermeiros que prestavam assistência na UCO participaram da implementação do HEP (Anexo 1), aplicado a setenta e cinco pacientes internados nesta unidade, no período de Janeiro a Maio de 1993.

Anteriormente à implementação do ICDP houve uma reunião na qual o instrumento foi apresentado, com esclarecimento de como seus itens e subitens deveriam ser preenchidos. Esta apresentação fez parte de um programa de reciclagem de todos os enfermeiros da unidade sobre a sistematização da assistência de enfermagem e foi realizada junto a pequenos subgrupos constituídos de 2 a 3 enfermeiros. Foi também orientado que o instrumento deveria ser preenchido por ocasião da admissão do paciente na unidade ou até 24 horas após.

O ICDP consistiu de seis itens: Identificação, Queixa do Paciente (Pregressa e Atual), Fatores de Risco/Antecedentes, Contra-Indicações para Trombólise, Exame Físico (de acordo com os sistemas orgânicos: Estado Geral, Sistema 
Neurológico, Sistema Respiratório, Sistema Cardiovascular, Sistema Gênito-urinário e Sistema Digestivo) e Informações Adicionais. Cada item foi subdividido de forma a especificar e organizar as informações obtidas.

Considerando-se o ambiente da OCO, onde o tempo acaba tornando-se um fator limitante para muitas atividades, procuramos desenvolver um instrumento que fosse composto por questões fechadas (tipo check-list), para agilizar o processo de coleta de dados, mas também por questões abertas, visando preservar a criatividade do enfermeiro na obtenção de dados.

Com a finalidade de avaliar a aplicabilidade do ICDP, foi utilizado um questionário composto por questões abertas e fechadas, referentes a cada item e sub-item do ICDP. (Anexo 2)

\section{RESULTADOS E DISCUSSÃO}

O questionário foi entregue aos onze enfermeiros que participaram da implementação do ICDP, sendo que um não respondeu. Analisando-se o ICDP quanto ao conteúdo, espaço para registro e distribuição, e levando-se em conta sua divisão nos itens: Identificação, Fatores de Risco, Contra-indicações para Trombólise, Exame Físico (e seus subitens), e Informações Adicionais, a maioria dos enfermeiros o considerou completo quanto ao conteúdo, com espaço suficiente para registro e distribuído adequadamente.

Foram sugeridas, entretanto, algumas modificações nos itens: Identificação, Fatores de Risco e Contra-indicações para Trombólise.

Segue-se a análise dos resultados obtidos em cada item:

\section{Identificação}

Foi sugerido o acréscimo dos dados: religião, número de filhos, horário de admissão e endereço residencial do paciente.

Já que o item Identificação presta-se ao levantamento de dados preliminares que irão ajudar o enfermeiro a determinar a identidade do paciente, podemos considerar como importante a informação quanto a religião e o número de filhos. Também o horário de admissão torna-se de grande valia na evolução do tratamento de alguns pacientes, principalmente daqueles com diagnóstico médico de infarto agudo do miocárdio. Na admissão do paciente na instituição, discrimina-se em um impresso próprio, que consta de seu prontuário, dados sócio-econômicos e seu endereço residencial. Sendo assim, não consideramos importante incluir estas informações no ICD, onde já existem dados como procedência e naturalidade, cuja 
interpretação epidemiológica, acreditamos, repercute de maneira mais relevante para a enfermagem (Quadro 1).

\section{QUADRO 1 - ITEM I (IDENTIFICAÇÃO) MODIFICADO}

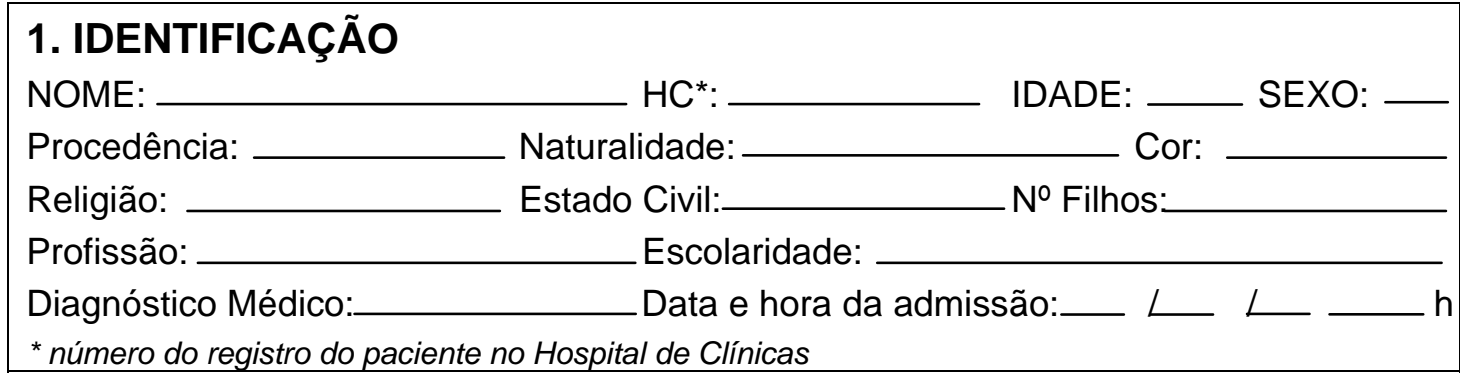

\section{Queixa do paciente}

Em relação a este item, $90 \%$ dos enfermeiros considerou importante sua subdivisão em queixa pregressa e atual. Nenhum deles sugeriu sua exclusão. A subdivisão deste item teve o propósito didático de evidenciar o quadro atual que levou o paciente a hospitalização e que pode, eventualmente, diferir das manifestações prévias da mesma patologia. Esses dados podem ser importantes tanto nas decisões da assistência de enfermagem imediata, quanto no processo de orientação do paciente em relação a sua doença, a fim de esclarecê-lo, por exemplo, que um distúrbio como a coronariopatia isquêmica pode manifestar-se como um quadro anginoso, reversível ou evoluir para um infarto agudo do miocárdio.

\section{Fatores de riscolantecedentes}

Neste item, alguns dos enfermeiros entrevistados sugeriram a inclusão do dado infarto do miocárdio prévio. Considerando-se a definição de fator de risco como sendo "qualquer hábito ou tratamento que possa ser utilizado para predizer a probabilidade de um indivíduo de desenvolver determinado distúrbio"16, e de acordo com o detalhamento cada vez mais preciso desses fatores na literatura, acreditamos ser muito importante, além do acréscimo do dado sugerido pelos enfermeiros, a ampliação, no ICD definitivo, do leque de caracterização dos seguintes fatores de risco: - Tabagismo: especificando se atual ou pregresso, quantidade e período; - Diabetes mellitus: se o paciente é ou não insulino-dependente ou faz uso de hipoglicemiantes orais; - Hipercolesterolemia: seria melhor designado como dislipidemias que, por sua vez, seria subdividido em hipercolesterolemia e hipertrigliceridemia; - Sedentarismo: seria acrescido de seu contra-ponto atividade física, especificada quanto ao tipo e freqüência; - Menopausa: especificada quanto a natural / cirúrgica, cursando ou não com reposição hormonal de estrógeno; - Álcool e Contraceptivos orais seriam acrescentados, especificando-se quantidade e período de uso. 
Em relação a Fatores de riscos não passíveis de modificação e/ou Antecedentes, acrescentaríamos o subitem hereditariedade, caracterizando-a quanto ao grau de parentesco, se consangüíneo direto ou indireto (Quadro 2).

\section{QUADRO 2 ITEM II (FATORES DE RISCO I ANTECEDENTES MODIFICADO}

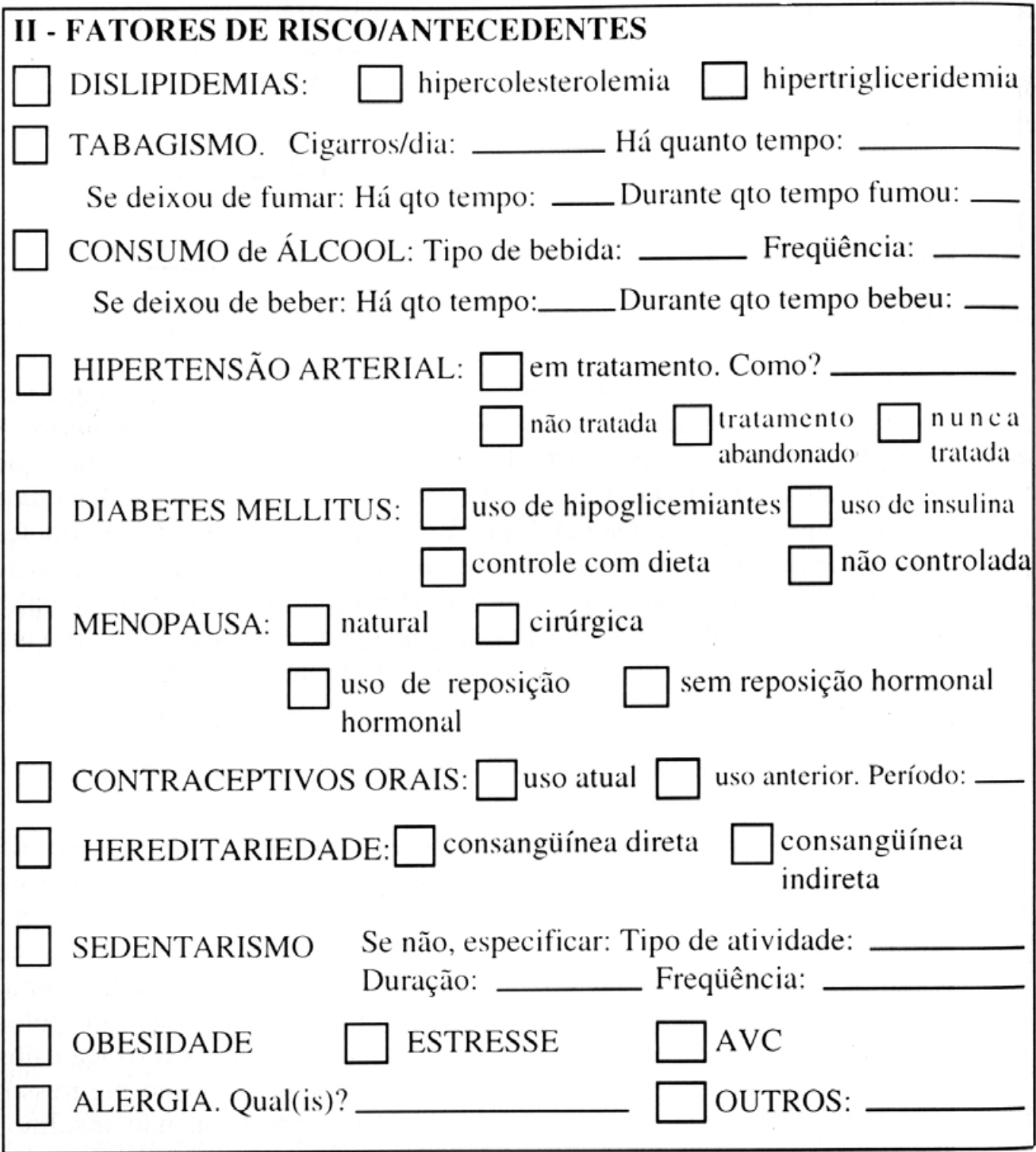

\section{Contra-indicações para trombólise}

Foi proposto pelos enfermeiros, acrescentar-se um espaço ao lado da contra-indicação para sua especificação mais detalhada, quando necessário (Quadro 3). 


\section{QUADRO 3 - ITEM III (CONTRA-INDICAÇÕES PARA TROMBÓLISE) MODIFICADO}

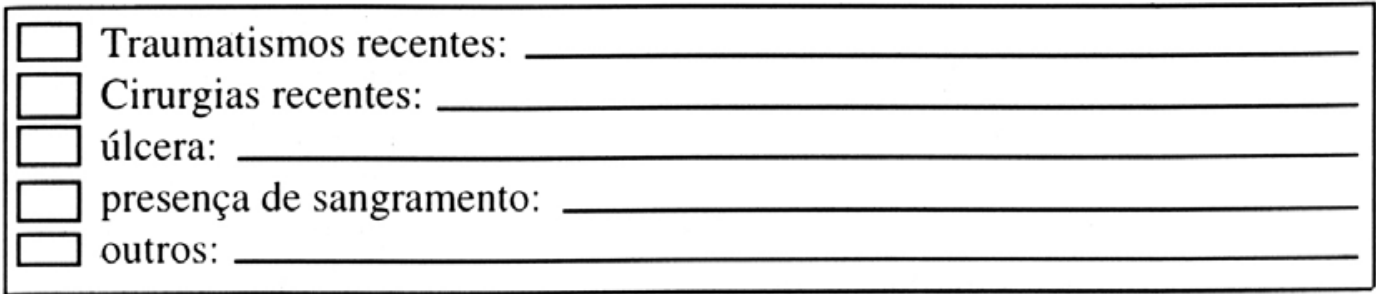

\section{Exame físico}

Todos os enfermeiros foram unânimes em concordar com a realização do levantamento de dados de acordo com os sistemas orgânicos. É importante ressaltar que, em nossa unidade, trabalhamos com os chamados pacientes críticos, que se caracterizam pela labilidade do equilíbrio entre seus sistemas orgânicos. Esse equilíbrio tão frágil pode ser comparado a "um tênue fio que separa a vida da morte"10. Nestes pacientes o funcionamento adequado dos vários sistemas orgânicos é fundamental para uma boa evolução do quadro, esperando-se da equipe de enfermagem não apenas a capacidade de ação rápida frente a uma situação de urgência, como também a vigilância preventiva constante, além de cuidados profiláticos a fim de evitar situações limítrofes.

O fato de optarmos pela realização do exame físico de acordo com os sistemas orgânicos deu-se pela preocupação em facilitar o levantamento de dados, tomando-o mais objetivo e preciso, utilizando-se uma abordagem que nos permitisse, ao longo da permanência do paciente na unidade coronária, a reavaliação constante dos principais parâmetros fisiológicos e, conseqüentemente, a conduta mais adequada para cada situação. E provável que, com a mesma preocupação, os enfermeiros tenham optado por este tipo de levantamento de dados.

O item Exame Físico foi subdividido em: Estado Geral, Sistema Neurológico, Sistema Cardiovascular, Sistema Gênito-urinário e Sistema Digestivo, cuja discussão dos resultados obtidos está descrita abaixo:

\section{- Estado geral}

Cinqüenta por cento dos enfermeiros responderam não ser viável a coleta de dados referentes a medidas antropométricas do paciente, devido a instabilidade de seu quadro clínico e a necessidade de repouso absoluto na ocasião da admissão.

Contudo, ao considerarmos a relevância destas medidas antropométricas para a realização do exame de cineangiocoronarioventriculografia realizado, com freqüência, no decorrer da internação do paciente na UCO, continuamos a sugerir que seria muito importante este levantamento assim que possível e mesmo que obtido verbalmente, tendo-se em conta, principalmente, a eventual necessidade de um estudo hemodinâmico de urgência. 


\section{- Sistema cardiovascular}

Todos os enfermeiros concordaram com a utilização de ilustrações para a descrição de pulsos e presença de cateteres. No paciente cardiopata, em especial naquele com coronariopatia, a avaliação cuidadosa dos pulsos arteriais periféricos é extremamente importante. A coronariopatia decorrente da aterogênese - um processo degenerativo, multifatorial em sua origem, que leva a um desajuste entre as três camadas que compõem a parede arterial ${ }^{15}$ - pode coexistir com o comprometimento simultâneo de outras artérias. Não é raro um paciente coronariopata apresentar alterações nos principais pulsos arteriais: carotídeo, braquial, radial, femoral, etc. Além disto, estes pacientes já foram ou serão submetidos a um estudo hemodinâmico (aquele cuja abordagem se faz por via arterial) o que também pode levar a modificações dos pulsos arteriais.

Acreditamos que o uso de ilustração (para a descrição dos pulsos) facilita a visualização, bem como o preenchimento deste dado. Por outro lado, a utilização do "boneco" para descrição dos cateteres, que tinha como finalidade permitir sua localização e via de inserção, talvez não seja muito adequada, devido a grande rotatividade dos cateteres. O mais apropriado, talvez, fosse manter o item em aberto para a descrição dos cateteres já instalados no momento da admissão.

A maioria dos enfermeiros referiu obstáculos no preenchimento dos subitens ECG e cateterismo cardíaco (60 e $80 \%$ respectivamente), alegando falta de conhecimento sobre eletrocardiograma e dificuldade em obter o resultado do cateterismo Junto ao paciente ou em seu prontuário.

Lembramos aqui, a relevância da autonomia de ação do enfermeiro em especial na UCO $^{18}$ (WHIPPLE et al., 1980). O conhecimento do ritmo normal e de seus principais distúrbios, bem como das alterações eletrocardiográficas que evidenciam isquemia miocárdica, são importantes e indispensáveis ferramentas para o trabalho do enfermeiro. Sendo este o profissional que permanece vinte e quatro horas ao lado do paciente, deve ter competência para detectar as alterações descritas anteriormente e tomar as condutas adequadas, em tempo hábil. Desta forma, acreditamos que a reciclagem do profissional que atua nesse campo deva ser feita de maneira contínua e abrangente, fornecendo elementos que subsidiem sua atuação, incluindo o conhecimento sobre exames diagnósticos específicos.

As informações relativas ao cateterismo cardíaco são importantes para que ele tome conhecimento, principalmente, das artérias comprometidas e da função ventricular, afim de que possa compreender o tratamento e sua evolução ${ }^{3}$. Isto reforça a necessidade de se levantar tais dados junto ao paciente/família/prontuário e equipe médica, para que o enfermeiro possa direcionar melhor sua assistência.

\section{- Sistema gênito-urinário}

Cinqüenta por cento dos respondentes referiu dificuldade no levantamento de dados referentes à genitália externa. Alguns alegaram constrangimento próprio (20\%), mas a grande maioria (80\%) constrangimento do paciente. Ressaltamos, 
porém, a importância do levantamento de todos os dados que possam determinar cuidados de enfermagem e, conseqüentemente, ajudar o paciente como indivíduo em sua totalidade, a restabelecer seu equilíbrio no ciclo saúde-doença.

Não houve críticas e/ou sugestões quanto a abordagem dos sistemas neurológico, respiratório e digestivo.

\section{INFORMAÇÕES ADICIONAIS}

Analisando-se os subitens expectativa/dúvidas do paciente/família em relação a sua doença, internação e tratamento, quase a totalidade dos enfermeiros considerou importante sua inclusão no ICDP, embora 60 e $80 \%$ tenham citado não ser possível a coleta de dados referentes a paciente e família, respectivamente. De acordo com 50\% estes dados seriam de grande valia para fornecer subsídios à uma melhor assistência de enfermagem. Um destes ressaltou, porém, que o enfermeiro não está preparado para coletar estas informações.

É necessário retomarmos aqui o contexto no qual foi realizado este trabalho: uma unidade coronária. O que leva o profissional de saúde a optar por esta área? Talvez o fascínio de ser uma ferramenta a trazer à vida alguém que se encontra tão próximo a morte? Ou trabalhar sempre em situações que exijam, atitudes rápidas, associadas a um raciocínio lógico e preciso? Ou no campo profissional, crescer cientificamente, quanto ao conhecimento de fisiopatologias complexas, adestramento no uso de aparelhos e equipamentos de última geração? Enfim, qualquer uma dessas motivações, pode levar o profissional a trabalhar numa unidade de cuidados intensivos.

Porém não se pode esquecer que o ponto central de trabalho de toda a equipe de saúde (não só de enfermagem) é o paciente - um ser único, que deve ser percebido e, conseqüentemente, cuidado em sua totalidade - nos aspectos bio-psico-sócioespiritual. Um indivíduo visto não apenas como um conjunto de sistemas orgânicos, cuja homeostase encontra-se comprometida, mas como uma pessoa com suas percepções, medos e ansiedades e que faz parte de um contexto sócio-cultural. Portanto, o compromisso do enfermeiro é amplo, significando o "cuidado do ser".

ARRUDA et al. ${ }^{2}$ discorrendo a respeito de pesquisa em enfermagem, comentam que, "o fascínio com o método e o afã de reconhecimento de foro de cientificidade nos leva, enfermeiros, a caminharmos em torno do método e nele nos ancorarmos, ao invés de utilizá-lo como adequado veículo ou instrumento para as descobertas pretendidas".

A partir desta idéia, poderíamos dizer que, atraídos pela sofisticação 
tecnológica temos nos distanciado, progressivamente, do cuidado global do paciente, privando-nos, cada vez mais, do privilégio que reside em cuidá-lo como um ser e das descobertas decorrentes deste processo. Perceber o paciente como um ser único, com necessidades específicas, buscando a maneira de atendê-las o mais adequadamente, faz parte de um "processo criativo, ou ato criador" o que, segundo ARRUDA et $\mathrm{al}^{2}$, traduz-se no "ânimo da enfermagem".

Voltando às respostas que nos levaram a esta reflexão notamos que, apesar de considerarem importante a obtenção destas informações, os enfermeiros alegaram não conseguir, no prazo de vinte quatro horas, levantar junto ao paciente e à família, os dados relacionados às suas expectativas quanto a doença, internação e tratamento, fato que foi atribuído a "falta de preparo" do profissional. Os motivos que levaram a estas respostas, talvez sejam aqueles já descritos. É possível, também, que o enfermeiro esteja mais desabituado do que despreparado para coletar tais informações.

Consideramos, porém, que ao elaborarmos o ICDP, também nós possamos ter visto estas informações sob um ângulo distorcido, quando as agrupamos sob o título de Informações Adicionais. Na verdade, não se trata de dados cujo levantamento é facultativo, ao contrário, são cruciais para a elaboração de um planejamento de assistência individualizada. Talvez fosse mais adequado designar este item de acordo com as informações que se propõe a levantar, como por exemplo: Levantamento das Condições Psicológicas e Sociais, como sugerido por BRADDY $^{5}$.

Assim, mais do que a mudança de roteiro de ICD acreditamos que, se faz necessária a renovação da própria mentalidade da equipe de enfermagem. Desta maneira, o instrumento de coleta de dados e a Metodologia da Assistência não podem ser utilizados como um fim em si mesmo, mas como forma de se atingir um determinado tipo de assistência de enfermagem, que seja aspirada pelo grupo como um todo.

Neste item foi avaliada, ainda, a opinião dos enfermeiros quanto a inclusão do levantamento das condições do paciente, para o auto-cuidado após a alta. Consideraram-no adequado $60 \%$ dos enfermeiros, alegando ser um dado importante para o planejamento e orientação quanto aos cuidados após a alta; $40 \%$ porém, salientou ter dificuldades relativas ao levantamento desse dado, citando-o como impróprio para o ICD. Alguns alegaram, também, que o autocuidado não caberia numa unidade de tratamento intensivo, sendo mais condizente à enfermaria.

O que provavelmente ocorre, é que dado o elevado grau de dependência que o paciente apresenta, principalmente nos primeiros dias de internação na UCO, acabamos por considerá-lo, em meio a rotina de nossas atividades, como mero "receptor" de cuidados. DANIEL ${ }^{8}$ descreve que, uma vez "sob nossa responsabilidade", esperamos que esse indivíduo tome-se de uma hora para outra, um paciente "por quem tudo se faz" ou que age exatamente como outras pessoas determinam. 
Porém, se o que procuramos, na realidade, é ajudá-lo a restabelecer seu equilíbrio, é fundamental que consigamos enxergá-lo como um indivíduo, participador ativo do "processo terapêutico".

Quando inserimos este sub-item no ICD preocupávamo-nos, exatamente, com a postura do paciente em relação ao seu estado de saúde, acreditando ser possível desenvolver, ainda em sua permanência na UCO, algum grau de auto-cuidado, ou pelo menos começar a direcioná-lo de modo que ele se tornasse capaz, mais tarde, de desenvolver-se neste sentido.

Dentre os enfermeiros, $70 \%$ sugeriu o levantamento de informações como: grau de ansiedade, comportamento frente ao enfermeiro e equipe de saúde, no sub-item: Impressões do Entrevistador, as quais serviriam para nortear o planejamento e a execução dos cuidados de enfermagem; $20 \%$ considerou este sub-item não relevante, uma vez que mostraria dados muito subjetivos.

De acordo com WALDOW ${ }^{17}$, a entrevista no momento do HE deve ser realizada com atenção e cuidado, pois é, neste momento, que se estabelece o início do relacionamento terapêutico. Segundo BRUNNER \& SUDDARTH ${ }^{6}$ o enfermeiro, nesta fase deve observar com olhos "de ver", ouvir com ouvidos "de ouvir", sentir com mãos "de sentir", para melhor interpretar os dados da entrevista/exame físico.

A capacidade do enfermeiro de proporcionar ao paciente uma participação ativa em seu atendimento não aparece espontaneamente: é preciso interesse e disposição, no sentido de procurá-lo como indivíduo, favorecendo-lhe a liberdade de expressão verbal, atentando, também, ao comportamento não verbal, que pode ser demonstrado de maneiras mais sutis, como gestos, postura e expressão facial ${ }^{6}$.

Esta capacidade de percepção acerca do paciente como indivíduo é talvez a menos desenvolvida, ou melhor, a mais atrofiada de nossas atividades. PRICE ${ }^{14}$; GOODALL $^{11}$ discutindo sobre o estabelecimento de rótulos e atribuição de características advindas deste rótulo ao paciente, comentam a facilidade com a qual, nós, profissionais de saúde, passamos a tratar os pacientes como "coronarianos", "deprimidos" ou ainda como diagnósticos médicos, "aquela angina", "aquela isquemia transitória", etc.

Dos enfermeiros, $80 \%$ considerou o ICDP bem direcionado a pacientes cardiopatas. Um enfermeiro (10\%) destacou a importância de se acrescentar aspectos sócio-econômicos e hábitos de vida.

Todos julgaram necessário o uso de um instrumento para a realização do ICD, tanto para organização quanto para a padronização das informações a serem colhidas, isto, segundo eles, repercutiria no melhor planejamento da assistência de enfermagem.

Curiosamente, porém, quando indagamos se o ICDP forneceu subsídios para melhorar a assistência de enfermagem, apenas 70\% julgaram-no importante como auxiliar no levantamento de problemas pertinentes à enfermagem, além de servir como instrumento de comunicação entre os enfermeiros. Enquanto $10 \%$ relatou não sentir dificuldade em prestar assistência sem o ICDP, 20\% dos profissionais questionados não respondeu. 
Reunimos aqui, algumas observações pertinentes a serem feitas em relação ao HE e a Metodologia da Assistência como um todo. HORTA ${ }^{12}$; DANIEL ${ }^{8}$; GOMES et al. ${ }^{9}$ entre outros, sugerem a importância do direcionamento do ICD para cada realidade onde será aplicado; motivo pelo qual, procuramos desenvolver um modelo que fosse peculiar para pacientes internados na UCO do HC-UNICAMP.

Historicamente, a utilização da Metodologia da Assistência tem levado a amplas discussões. São muitos os autores que descrevem as divergências de opinião sobre sua aplicabilidade na prática. Seu desenvolvimento e implementação são defendidos, geralmente, pelos que o tomam como decorrência de uma busca de melhoria do trabalho de enfermagem, através de uma metodologia científica. As dificuldades na utilização do método ou suas limitações constituem, por outro lado, o grande alvo para as crítlcas negativas ${ }^{13}$.

Valeria a pena frisar, mais uma vez, a importância do uso da metodologia, seja qual for a teoria na qual se baseia, como um meio e não como meta final, para que possamos implementar a ideologia que norteará o trabalho da equipe de enfermagem. De acordo com a filosofia de nossa instituição, o uso da metodologia da assistência deveria nos auxiliar a perceber o paciente como um indivíduo em sua totalidade, reconhecer nele os problemas passíveis de ação de enfermagem, e assim ajudá-lo a restabelecer seu equilíbrio.

As respostas que obtivemos em relação à necessidade do uso de um instrumento de ICD e sua repercussão na assistência de enfermagem, ressaltam a diferença que existe entre a ideologia, que leva todos a defenderem sua utilização, e a prática, quando há quem não o considere fundamental no atendimento ao paciente, Talvez mais do que um problema inerente ao instrumento e/ou a metodologia de assistência, exista nos profissionais uma incorporação tão forte da rotina de seu serviço na realização de tarefas, que acaba por limitá-lo na percepção dos problemas que o paciente apresenta, ou a levá-lo a levantar dados que são simplesmente colhidos, mas não devidamente analisados. Talvez, também, porque não fossem pertinentes à enfermagem, ou porque não tenham recebido a devida importância e, conseqüentemente, não tenham sido alvos do "cuidar"4,15.

Quanto ao tempo utilizado para o preenchimento de ICDP, foi citado um intervalo entre dez e quarenta minutos por $70 \%$ dos enfermeiros. Destes, alguns esclareceram que para seu preenchimento completo poderiam ser utilizadas até vinte e quatro horas; $30 \%$ não respondeu. Quanto ao tempo ideal para preenchimento foi considerado de quinze a trinta minutos (60\%), três horas (10\%), de doze até vinte e quatro (10\%); $20 \%$ não respondeu. Portanto, de acordo com a maioria dos enfermeiros o tempo referido como ideal foi coincidente com o tempo empregado para a entrevista e o exame físico. Quando propusemos um modelo de ICD para UCO, preocupávamo-nos em encontrar um meio de coletar o máximo de informações para a enfermagem, no menor tempo possível, considerando, sempre, o ambiente tumultuado e corrido neste setor. Porém, o momento em que se realiza o ICD é quando se inicia também o relacionamento interpessoal, enfermeiro-paciente; daí a preocupação em utilizar, dentro da disponibilidade, o tempo que for necessário para o desenrolar desta interdependência. 
Outro fator considerável, lembrado por poucos enfermeiros, é que a coleta de dados pode ser completada dentro das vinte e quatro horas que se seguem à admissão do paciente e deve ser atualizada sempre que necessária durante o período de internação do indivíduo.

\section{CONCLUSÕES}

Dos resultados obtidos com a realização deste trabalho, concluímos que: 1. 0 desenvolvimento e a implementação de um instrumento para coleta de dados foram considerados válidos pela maioria dos enfermeiros. 2. quase a totalidade dos entrevistados considerou o ICDP completo, com distribuição adequada de itens e subitens e voltado para pacientes cardiopatas. 3. as modificações sugeridas no ICDP, pelos enfermeiros, e a avaliação de seu preenchimento pelas autoras, servirão como subsídio na reelaboração do ICDP e no desenvolvimento do instrumento definitivo de ICD para a UCO do NAMI do HC-UNICAMP.

\section{NURSING ASSESSMENT: A PILOT MODEL STUDY}

The overall purpose of this study was to explore nurses' feelings about the applicability and adequacy of a pilot model of nursing assessment (PMNA) developed for coronary care units (CCU) in order to obtain data that could help in establishing a definitive model. The evaluation. performed by $11 \mathrm{CCU}$ nurses, showed that they considered the development and implementation of PMNA as valuable, and that its design was adequate for interviewing cardiac patients. These results will be employed in the elaboration of a definitive model of nursing assessment.

UNITERMS: nursing assessment, coronary care units

\section{LEVANTAMIENTO DE DATOS: EVALUACIÓN DE UN MODELO PILOTO}

Con la elaboración e implementación de un modelo piloto del instrumento de Levantamiento de Dados (LDP) para la Unidad Coronária (UCO), tu vimos como objetivo evaluar las consideraciones de los enfermeros sobre la aplicabilidad del HEP y su 
adecuación para la UCO, así como también levantar elementos que contribuyon en su elaboración. La evaluación realizada a través de un cuestionario que fue entregado a once enfermeros que participaron en La implementación del HEP, nos mostró que ellos consideran valederos tanto La elaboración como La implementación del HEP, interpretado como adecuado en forma y contenido para pacientes cardiópatas, además de dar ayudas para La elaboración de un instrumento definitivo del HEP,

TÉRMINOS CLAVES: coronariopatía, enfermería, atención al paciente

\section{REFERÊNCIAS BIBLIOGRÁFICAS}

01. ANSELMI, M.L.; CARVALHO, E.C.; ANGERAMI, E.L.S. Histórico de enfermagem: compreensão e utilização técnica - prática. Rev.Esc.Enfermagem USP, v. 22, n. 2, p. 181- 188, 1988.

02, ARRUDA, E. N.; DIAS, L.P.M.; SILVA, A.L. Pesquisar para assistir. Rev.Esc. Enfermagem USP, v. 26, p. 119 - 124, 1992. Número especial: Encontro Internacional "Pesquisa em Enfermagem: uma questão de Saúde".

03. BEATTU, S.et al. The use of cardiac catheterization data to design nursing care plans. Crit.Care Nurse, v. 10, n. 6, p. 43-53, 1990.

04. BOEHS, A.E,; PATRICIO, Z,M, O que é este "cuidar/cuidado"?: uma abordagem inicial. Rev .Esc.Enfermagem USP, v. 24, n. 1, p. 111-6, 1990.

05. BRADDY, P,K. Assessment tool. Crit. Care Nurse, v. 9, n. 9, p. 71-81, 1989.

06. BRUNNER, L.S.; SUDDARTH, D,S. Tratado de enfermagem médico-cirúrgica, São Paulo: Interamericana, 1982. v. 1.

07. CASTELLANOS, B. E. P.; CASTILHO, V. Marco conceitual da assistência de enfermagem: considerações gerais. In: CAMPEDELLI, M. Cetal. Processo de enfermagem na prática. São Paulo: Ática, 1989.

08. DANIEL, L.F. A enfermagem planejada. 3.ed. São Paulo: E.P.U., 1981

09. GOMES, G.B. et al. Aplicação do processo de enfermagem a pacientes inconscientes internados na UTI de um hospital de Curitiba.

Rev.Paul.Enfermagem, v. 7, p. 22-27, 1987.

10. GONÇALVES Jr, F.L. Prefácio. In: TERZI, R \& ARAÚJO, S. Técnicas básicas em U.T.I. Campinas: UNICAMP, 1988.

11. GOODALL, C. How do we teach the nursing process? Nurs. Times, v. 84 , n. 48, p. 47-49, 1988.

12. HORTA, W.A. Processo de enfermagem. São Paulo: E.P.U., 1979.

13. McHUGH. Does the nursing process reflect quality care assessment tool. Crit.CareNurse, v. 11, n. 6, p. 32,1991. 
14. PRICE, B. J. First impressions: paradigms for patient assessment. J. Adv .Nurs. v. 12, n. 6, p. 699-705, 1987.

15. ROSS, R. T. The pathogenesis os atherosclerosis. In: BRAUNW ALD, E. Heart disease: a textbook of cardiovascular disease. 3. ed. New York: W. B. Saunders, 1988.

16. RULLER, L. H. Controlling coronary heart disease: where he will stand by the end of this decade (Persp. Lip. Dis. [In press]) Apud ROSS, R. T. The pathogenesis of atherosclerosis. In: BRAUNW ALD, E. Heart disease. A textbook of cardiovascular medicine. 3. ed. New York: W. B. Saunders, 1988.

17. WALDOW, V. R. Processo de enfermagem: teoria e prática. Rev. Gaúcha Enfermagem, v. 9, n. 1, p. 14-22, 1988.

18. WHIPPLE, G. H. et al. Insuficiência coronariana assistência e tratamento. São Paulo: E.P.U., 1980. 


\section{ANEXO 1 \\ HOSPITAL DE CLÍNICAS - NÚCLEO DE ASSISTÊNCIA MÉDICA \\ INTENSIVA UNIDADE CORONÁRIA SISTEMATIZAÇÃO DA ASSISTÊNCIA D ENFERMAGEM INSTRUMENTO DE COLETA DE \\ DADOS - PILOTO}

\section{IDENTIFICAÇÃO}

NOME:

$\mathrm{HC}$ :

IDADE:

Procedência:

Naturalidade:

SEXO:

Profissão: Escolaridade:

Cor:

Diagnóstico:

Data de admissão:

\section{QUEIXA DO PACIENTE}

Atual:

Pregressa:

\section{FATORES DE RISCO/ANTECEDENTES}

$\square$ hipertensão arterial $\square$ tabagismo $\square$ diabetes $\square$ obesidade $\square$ AVC $\square$ estresse $\square$ sedentarismo $\square$ hereditariedade $\square$ hipercolesterolemia $\square$ menopausa $\square$ alergias $\square$ outros. Qual(is):

Uso de medicamentos:

\section{CONTRA-INDICAÇÕES PARA TROMBÓLISE}

$\square$ traumatismos recentes $\square$ cirurgias recentes $\square$ úlcera $\square$ presença de sangramento $\square$ coagulopatia $\square$ outros. Qual(is):

\section{EXAME FÍSICO}

\section{Estado Geral}

Hidratação: coloração de mucosas:

temperatura axilar: peso: altura:

condições de higiene:

integridade cutâneo-mucosa:

sistema músculo-esquelético:

\section{Sistema Neurológico}

Nível de consciência: pupilas: grau de orientação: déficits: $\square$ motor qual(is): outros:

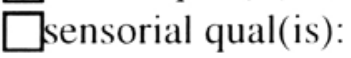




\section{Sistema Respiratório}

Respiração: $\square$ espontânea $\square$ assistida. Tipo:

FR: Pådrão respiratório:

Ausculta pulmonar:

Outros (tosse/expectoração; drenos/tubos):

\section{Sistema cardiovascular}

FC: Ritmo:

Se em uso de drogas, especificar:

ECG:

PA: Se em uso de drogas, especificar:

Pulsos:

$(+)$ presente

( \pm ) presente, filiforme

(-) ausente

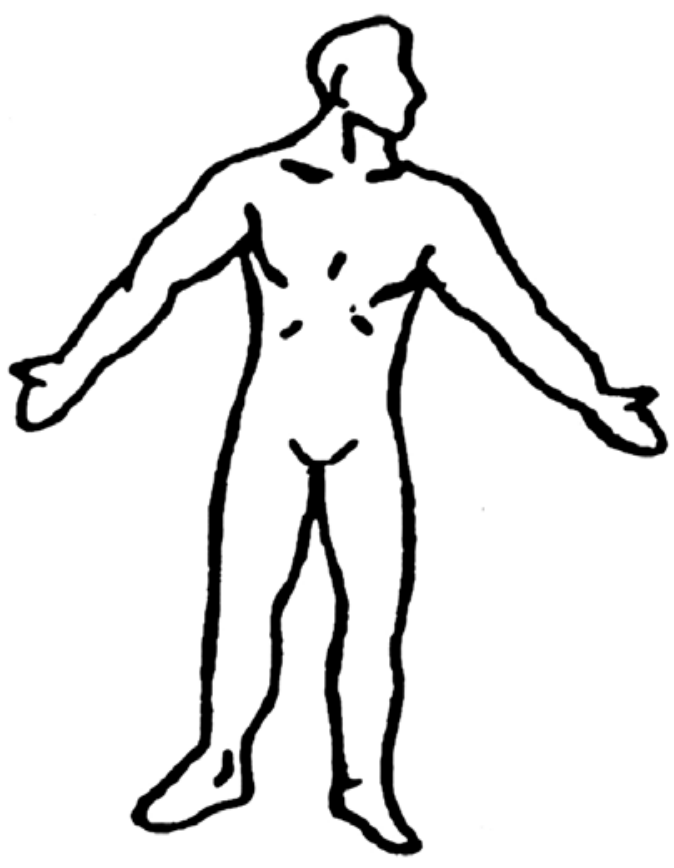

\section{Cateterismo prévio:}

Rede Venosa:

Condições para venóclise: $\square$ boas $\square$ regulares $\square$ más Estase jugular: varicosidades:

Queixas (descrever): precordialgia, dispnéia, edema, claudicação, lipotímia: 
Presença de catéteres:

(IC) Intracath (FE) Flebotomia (PA) Pressão Arterial média (SG) Swan-Ganz (MP) marca-passo

(VS) venóclise-scalp (VA) abocath

\section{Sistema Gênito-Urinário}

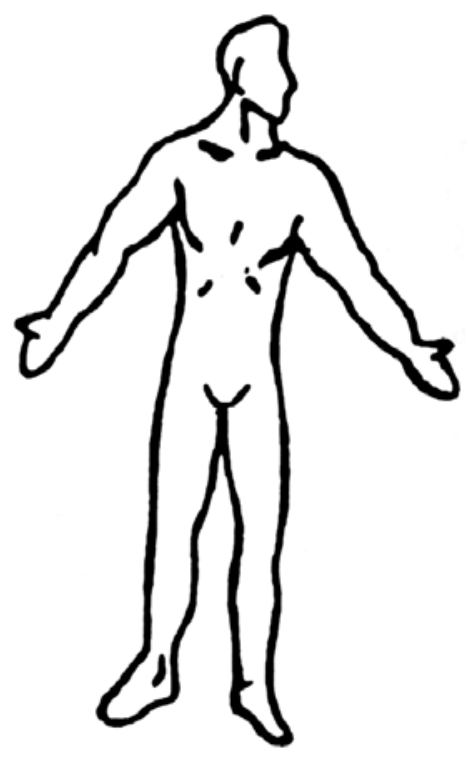

Genitália externa:

Hábito urinário (freqüência, aspecto):

Queixas:

Outros: (sondas/bexigoma):

\section{Sistema Digestivo}

Hábito intestinal: (freqüência, características):

Costume alimentar:

Queixas: (dor, náusea/vômitos, inapetência, hemorróidas)

Abdomen:

\section{INFORMAÇÕES ADICIONAIS}

Expectativas/dúvidas do paciente quanto a:

doença, internação, tratamento:

Expectativas/dúvidas da família (especificar o grau de parentesco) em relação a: doença internação e tratamento do paciente:

Condições para o cuidado pós-alta:

Impressões do entrevistador:

Informante:

Data: Enfermeiro:

COREn: 


\title{
ANEXO 2
}

\author{
INSTRUMENTO PARA AVALIAÇÃO DO MODELO PILOTO DE \\ INSTRUMENTO DE COLETA DE DADOS DA UNIDADE CORONÁRIA - \\ HC - UNICAMP
}

1. Analisando o item IDENTIFICAÇÃO, qual sua opinião sobre:

1.1. CONTEÚDO $\square$ completo $\square$ incompleto. Por que?

1.2. ESPAÇO PARA REGISTRO $\square$ suficiente $\square$ insuficiente. Especifique. 1.3. DISTRIBUIÇÃO DOS SUB-ÍTENS $\square$ adequada $\square$ inadequada. Por que?

2. Analisando o item QUEIXA DO PACIENTE, qual sua opinião sobre:

2.1. ESPAÇO PARA O REGISTRO \suficiente $\square$ insuficiente. Especifique. 2.2. Na sua opinião, qual a importância da sub-divisão deste item, em queixa pregressa e atual?

3. Analisando o item FATORES DE RISCO, qual sua opinião sobre:

3.1. CONTEÚDO $\square$ completo $\square$ incompleto. Por que?

3.2. ESPAÇO PARA REGISTRO $\square$ suficiente $\square$ insuficiente. Especifique 3.3. DISTRIBUIÇÃO DOS SUB-ITENS $\square$ adequada $\square$ inadequada. Por que?

4. Analisando o item CONTRA-INDICAÇÕES PARA TROMBÓLISE, qual sua opinião sobre:

4.1. CONTEÚDO $\square$ completo $\square$ incompleto. Por que?

4.2. ESPAÇO PARA REGISTRO $\square$ suficiente $\square$ insuficiente. Por que?

4.3. DISTRIBUIÇÃO DOS SUB-ITENS $\square$ adequada $\square$ inadequada. Por que?

5. Analisando o item EXAME FÍSICO, qual sua opinião sobre:

a) o levantamento de dados agrupado de acordo com os sistemas orgânicos

$\square$ adequado $\square$ inadequado. Justifique.

5.1. Analisando o sub-item ESTADO GERAL, qual sua opinião sobre:

5.1.1. CONTEÚDO $\square$ completo $\square$ incompleto. Por que?

5.1.2. ESPAÇO PARA REGISTRO $\square$ suficiente $\square$ insuficiente. Especifique.

5.1.3. DISTRIBUIÇÃO DOS SUB-ITENS $\square$ adequada $\square$ inadequada. Por que?

5.1.4. Você acha possível realizar as medidas antropométricas do paciente (peso/altura) nas primeiras 24 horas após a admissão do paciente? 
5.2. Analisando o sub-item SISTEMA NEUROLÓGICO, qual sua opinião sobre:

5.2.1. CONTEÚDO $\square$ completo $\square$ incompleto. Por que?

5.2.2. ESPAÇO PARA REGISTRO $\square$ suficiente $\square$ insuficiente. Especifique

5.2.3. DISTRIBUIÇÃO DOS SUB-ITENS $\square$ adequada $\square$ inadequada. Por que?

5.3. Analisando o sub-item SISTEMA RESPIRATÓRIO, qual sua opinião sobre:

5.3.1. CONTEÚDO $\square$ completo $\square$ incompleto. Por que?

5.3.2. ESPAÇO PARA REGISTRO $\square$ suficiente $\square$ insuficiente. Especifique

5.3.3. DISTRIBUIÇÃO DOS SUB-ITENS $\square$ adequada $\square$ inadequada. Por que?

5.4. Analisando o sub-item SISTEMA CARDIO-VASCULAR, qual sua opinião sobre:

5.4.1. CONTEÚdO $\square$ completo $\square$ incompleto. Por que?

5.4.2. ESPAÇO PARA REGISTRO $\square$ suficiente $\square$ insuficiente. Especifique

5.4.3. DISTRIBUIÇÃO DOS SUB-ITENS $\square$ adequada $\square$ inadequada. Por que?

5.4.4. Você encontrou alguma dificuldade no preenchimento do sub-item ECG?

$\square$ não $\square$ sim. Justifique

5.4.5. Você encontrou alguma dificuldade no preenchimento do sub-item cateterismo?

$\square$ não $\square$ sim. Justifique

5.4.6. Você julgou valida a utilização de ilustrações para identificar os subitens:

- PULSO: $\square$ sim $\square$ não. Por que?

- PRESENÇA DE CATÉTERES: $\square \operatorname{sim} \square$ não. Por que?

5.5. Analisando o SISTEMA GÊNITO-URINÁRIO, qual sua opinião sobre:

5.5.1 CONTEÚDO $\square$ completo $\square$ incompleto. Por que?

5.5.2. ESPAÇO PARA REGISTRO $\square$ suficiente $\square$ insuficiente. Por que? 5.5.3. DISTRIBUIÇÃO DOS SUB-ITENS $\square$ adequada $\square$ inadequada. Por que?

5.5.4. Você encontrou dificuldades no preenchimento do sub-item genitália externa?

$\square$ não $\square$ sim. Justifique 
5.6. Analisando o sub-item SISTEMA DIGESTIVO, qual sua opinião sobre: 5.6.1. CONTEÚDO $\square$ completo $\square$ incompleto. Por que?

5.6.2. ESPAÇO PARA REGISTRO $\square$ suficiente $\square$ insuficiente. Especifique

5.6.3. DISTRIBUIÇÃO DOS SUB-ITENS $\square$ adequada $\square$ inadequada. Por que?

6. Analisando o item INFORMAÇÕES ADICIONAIS, qual sua opinião quanto a: 6.1. CONTEÚDO $\square$ completo $\square$ incompleto. Por que?

6.2. ESPAÇO PARA REGISTRO $\square$ suficiente $\square$ insuficiente. Especifique 6.3. DISTRIBUIÇÃO DOS SUB-ITENS $\square$ adequada $\square$ inadequada. Por que?

6.4. Foi possível coletar dados referentes a expectativa do paciente em relação a:

SUA DOENÇA/INTERNAÇÃO/TRATAMENTO $\square \operatorname{sim} \square$ não. Justifique

6.5. Você considera importante a coleta destes dados?

6.6. Foi possível coletar dados junto a familia quanto a:

DOENÇA/INTERNAÇÃO/TRATAMENTO DO PACIENTE?

$\square \operatorname{sim} \square$ não. Justifique

6.7. Você considera adequado o levantamento das CONDIÇÕES PARA O AUTO-CUIDADO APÓS ALTA, no Histórico de Enfermagem?

6.8. O que você acha que deveria ser preenchido nos sub-itens IMPRESSÕES DO ENTREVISTADOR?

6.9. Qual a importância que você atribui a estes dados?

7. Quanto tempo em média, você utilizou para o preenchimento deste histórico?

8. Considerando-se o tipo de paciente internado, número de funcionários e de procedimentos na Unidade Coronária, quanto tempo você julga necessário para o preenchimento de um histórico de enfermagem?

9. Você julga necessário o uso de um instrumento para a realização do histórico de enfermagem?

10. O histórico forneceu subsídios para melhorar a qualidade da assistência de enfermagem prestada ao paciente internado na Unidade Coronária?

11. Na sua opinião, os dados levantados foram suficientes para caracterizar o paciente cardiopata? 